USC-94-006

cond-mat/9403095

\title{
Exact Theory of Polymer Adsorption in Analogy with the Kondo Problem
}

\author{
P. Fendley and H. Saleur* \\ Department of Physics, University of Southern California \\ Los Angeles CA 90089
}

\begin{abstract}
We conjecture the exact scaling theory for the adsorption of two-dimensional polymers by using boundary S matrices. We compute the boundary free energy (the "g-function"), study the flow from adsorbed to desorbed phase, and derive the crossover exponent and all the geometrical exponents at the transition. More generally, we solve the special transition in the $O(n)$ model, the polymer case corresponding to $n=0$. The $n=2$ limit appears identical to the ordinary Kondo problem.
\end{abstract}

* Packard Fellow

$3 / 94$ 
There has recently been a surge of activity in the study of two-dimensional field theories with boundaries. These are of importance in the many different contexts of open string theory, the Callan-Rubakov effect, solid-state physics, and dissipative quantum mechanics [1]. We point out in this letter that there is another interesting physical problem in this category: the adsorption of two-dimensional polymers. We solve it exactly, and find a remarkable analogy with the ordinary Kondo problem.

We study a long two-dimensional polymer in the presence of a boundary. This system can be realized experimentally with polymeric materials that spread reproducibly at an air-water interface [2]. The solvent is such that the polymer is in the universality class of lattice self-avoiding walks. We suppose the polymer has a short-range interaction with the boundary, which in the lattice model corresponds to a Boltzmann weight $e^{\epsilon^{s}}$ per adsorbed monomer. At small $\epsilon^{s}$ entropy dominates and the polymer is not adsorbed. As $\epsilon^{s}$ is increased, a critical point $\epsilon_{c}^{s}$ is reached where energy and entropy terms compensate. At this point, the number of adsorbed monomers varies with the total length $N$ as $N_{\text {ads }} \propto N^{\varphi}$, where $\varphi$ is some crossover exponent [3]. For $\epsilon^{s} \leq \epsilon_{c}^{s}$, the typical size of the polymer varies with the well-known two-dimensional bulk exponent: $\left\langle R^{2}>\propto N^{2 \nu}, \nu=3 / 4\right.$. For $\epsilon^{s}>\epsilon_{c}^{s}$, energy dominates and at large distance the polymer behaves like a one-dimensional object stuck to the wall, with in particular $<R^{2}>\propto N^{2}$. There is a finite fraction of adsorbed monomers which varies as $N_{\text {ads }} / N \propto\left(\epsilon^{s}-\epsilon_{c}^{s}\right)^{-1+1 / \varphi}$.

It is well known that polymers can be described by the geometrical $O(n)$ model as $n \rightarrow 0$ [3]. The adsorption transition is numerically known to occur for the loops of the $O(n)$ model at least for $n \in[0,1]$ (later we argue that this is true for $n \leq 2$ ), so we build a theory for it as a function of $n$. As a surface critical phenomena this adsorption transition can be considered as the special transition [4]. Although the boundary is one-dimensional, it can order for geometrical models because they are non-local and non-unitary. The special transition is not the only boundary critical point; there are also the ordinary and extraordinary transitions which correspond to free and fixed boundary conditions in the $O(n)$ model.

Following [5], we consider a model of self-avoiding and mutually-avoiding polymer loops on some lattice with fugacity $n$ per loop and $x_{c}$ per monomer. The first non-trivial order as $n \rightarrow 0$ is a single self-avoiding loop and gives the original problem. We choose the fugacity $x_{c}$ so that we are at the critical point in the bulk (this point does not depend on what happens at the boundary for $\epsilon^{s} \leq \epsilon_{c}^{s}$ ) and deal therefore with very long loops. 
Monomers adsorbed at the boundary have a different fugacity $x^{s} \equiv e^{\epsilon^{s}}$. Near the boundary fixed points the problem can be viewed at large distance as a conformal field theory (with the appropriate scale-invariant boundary conditions) perturbed by the energy operator on the boundary. At the free fixed point, this operator has surface scaling dimension 2 [6], while at the special point it has dimension $(m-1) /(m+1)$ [7], with $n=2 \cos \pi / m$. The operator is relevant at the special point and irrelevant at the free boundary, so it induces a flow from special to free.

We make the crucial assumption that the boundary does not destroy the integrability of the scaling limit of the critical $O(n)$ model. This is very likely since the conformal minimal models with $\Phi_{1,3}$ perturbation on the boundary are integrable $[\mathbb{8}$, and the $O(n)$ models with energy perturbation on the boundary share many properties with these models. We can then require the constraints of integrability, in particular the factorizability of the $S$ matrix.

To proceed further we make an analogy with the Kondo problem of a single species of electron $(k=1)$ coupled to a spin- $1 / 2$ impurity. As is well known, the $s$ waves in this three-dimensional nonrelativistic problem reduce to a relativistic $1+1$-dimensional problem on the half-line, with the impurity lying on the boundary. In the following we shall also treat the polymers as a $1+1$-dimensional problem by performing a Wick rotation. It is found (for reviews, see [9]) that there are two critical points in the Kondo problem. In the UV $(T \rightarrow \infty)$ the impurity is decoupled and therefore there is a spin $1 / 2$ sitting at the boundary, while in the IR $(T=0)$, the impurity is screened by a bound electron. The model interpolates between the two fixed points, with the Kondo temperature $T_{K}$ the scale at which the behavior crosses over from one critical point to another. This problem is conveniently approached using $S$ matrices [10]. 1 . In the bulk there is a doublet of leftmoving particles carrying a label 1,2 (which stands for $S_{z}= \pm \frac{1}{2}$ ), and likewise for the right. The particles are massless with dispersion relation $E= \pm p$ which we parametrize by $E=\mu e^{-\theta}$ for the left-movers and $E=\mu e^{\theta}$ for the right. All physics is independent of the arbitrary scale $\mu$. At the bulk critical point, right and left particles are independent (the two Fermi surfaces are infinitely far apart), so the scattering matrix $S_{L R}=1$. The bulk scattering matrix for two left movers with rapidities $\theta_{1}$ and $\theta_{2}$ reads

$$
S_{L L}=S_{R R}=Z(\theta)\left(I-\frac{\theta}{\theta-i \pi} e\right),
$$

1 We discuss only the spin degrees of freedom; since the charge degree of freedom does not couple to the boundary, we ignore it. 
where $\theta \equiv \theta_{1}-\theta_{2}, I$ is the identity matrix, and $e=K_{1}$, with

$$
K_{q}=\left(\begin{array}{cccc}
0 & 0 & 0 & 0 \\
0 & -1 & q^{-1} & 0 \\
0 & q & -1 & 0 \\
0 & 0 & 0 & 0
\end{array}\right)
$$

The function $Z(\theta)$ is a known factor ensuring unitarity and crossing symmetry. The impurity can be thought of as a single particle sitting at the boundary; the $S$ matrix for scattering a left mover off the impurity (so it becomes a right mover) is

$$
\left(S_{B L}\right)_{i_{1}}^{j_{1}}=-\delta_{i_{1}}^{j_{1}} i \tanh \left(\frac{\theta-\theta_{K}}{2}-\frac{i \pi}{4}\right),
$$

This is the simplest solution of the boundary "cross-unitarity" relation [8]. The "boundary rapidity" $\theta_{K}$ is related to $T_{K}$ by $\theta_{K} \equiv \ln \left(\mu / T_{K}\right)$.

The bulk $S$ matrix (1) for the Kondo problem is in fact the $S$ matrix for the critical $O(n)$ model at $n=2[11]$. We can extend this analogy to the boundary case and then to other values of $n$. Suppose we draw the trajectories of the Kondo particles in $1+1$-dimensional space as lines in the plane. The bulk S-matrix can be rewritten in the manifestly $O(2)$ symmetric form as

$$
\left(S_{L L}\right)_{i_{1} i_{2}}^{j_{1} j_{2}}=Z(\theta)\left[\delta_{i_{1}}^{j_{2}} \delta_{i_{2}}^{j_{1}}+f(\theta) \delta_{i_{1} i_{2}} \delta^{j_{2} j_{1}}\right]
$$

The three possible $O(n)$-invariant $S$ matrix elements are the three ways two lines can meet each other at a vertex. The absence of the third invariant tensor in (3) leaves only selfavoiding trajectories, and formally the resulting configurations are identical to the ones of the lattice $O(2)$ model. Since the Kondo boundary scattering is equivalent to that of a single particle sitting at the boundary, we get in this picture a "shadow" line on the wall. Its effect depends on the energy scale $T_{K}$; in the UV the line is there and behaves like an adsorbed line in the $O(2)$ lattice model, whereas in the IR it disappears. By this analogy we see that the Kondo flow, after appropriate change of variables, looks very much like the flow from special to free in the $O(2)$ model (we will make this statement mathematically precise soon). A natural idea is then to extend the known solution of the Kondo problem to $n$ species and then make a continuation to $n=0$ to solve our polymer problem.

How to do this in the bulk is well known. The S matrix (3) can obviously generalized to an $O(n)$ symmetric one by allowing $i$ and $j$ to run from 1 to $n$. We write it as

$$
S_{L L}=Z(\theta)\left[I+f(\theta) e^{(n)}\right]
$$


The function $f$ is determined by requiring that the $S$ matrix obeys the Yang-Baxter equation. The matrix $e$ in (1) satisfies the Temperley-Lieb algebra relations $T L(2)$ at the particular value $n=2$ of the usual parameter, while the $e^{(n)}$ matrices provide a representation of the $T L(n)$ algebra. Using this algebra, one finds that the Yang-Baxter equation reduces solely to a functional equation for $f(\theta)$ depending on $n$. The solution is [11]

$$
f(\theta)=\frac{\sinh (\theta / m)}{\sinh (\theta-i \pi) / m}
$$

where $n=q+q^{-1}$ and $q=\exp \frac{i \pi}{m}$. Physical predictions depend only on the algebra satisfied by the $S$ matrices and not the particular representation of the algebra [11, 12, 13]. We can thus use the other representation $e^{(n)}=K_{q}$. Because this representation makes sense for $n$ non-integer, it provides the desired analytic continuation. The boundary $S$ matrix is (2) in all of these representations.

To conclude: we conjecture that the bulk $\mathrm{S}$ matrix (4) with $e^{(n)}=K_{q}$ and the boundary $S$ matrix (2) describe the full field theory that interpolates between special and free boundary conditions for the $O(n)$ model with $n \leq 2$. This theory has massless bulk particles and a boundary $S$ matrix depending on a scale $T_{K}$.

To test this conjecture, we show that it gives a number of quantitative predictions for the special transition, all consistent with known results. We use the thermodynamic Bethe ansatz (TBA) to derive the free energy resulting from the boundary $S$ matrix. As usual, instead of looking at right and left movers on the half-line, we look at the equivalent problem of left movers on the full line. This transforms the boundary into a "particle" fixed at the origin. To quantize the momenta, instead of the full line we take space to be a circle of length $L$. We therefore consider the $O(n)$ model as a $1+1$-dimensional problem at quantum temperature $T$. Physically this corresponds to the statistical mechanical problem on a torus of length $L$ and circumference $R=\frac{1}{T}$, with one impurity line in the middle. There is a bulk free energy independent of the boundary coupling, as well as a surface or impurity free energy. To compute the latter we need to specify the value of $n$, and use a representation of the $S$ matrix algebra for this particular value. The simplest approach will be to make computations for $n=2 \cos (\pi / m)$ where $m$ is an integer, and then to continue naively the results to $m$ non-integer or $m=2$. The bulk calculation can be found, for example, by taking the zero-mass limit of the $O(n)$ model calculation in [13]. The free energy is given in terms of the pseudo-energies $\epsilon_{j}(\theta)$ obeying the set of integral equations

$$
\epsilon_{j}(\theta)=\delta_{j 1} e^{-\theta}-\int \frac{d \theta^{\prime}}{2 \pi} \frac{1}{\cosh \left(\theta-\theta^{\prime}\right)}\left(\ln \left(1+e^{-\epsilon_{j-1}\left(\theta^{\prime}\right)}\right)+\ln \left(1+e^{-\epsilon_{j+1}\left(\theta^{\prime}\right)}\right)\right),
$$


where $j=1, \ldots, m-2$ and $\epsilon_{0}=\epsilon_{m-1} \equiv \infty$. Including a non-trivial boundary $S$ matrix changes the quantization condition for the particles' momenta, which changes the density of states. This in turn adds an extra piece to the free energy, yielding

$$
f_{\mathrm{imp}}=-T \int \frac{d \theta}{2 \pi} \frac{1}{\cosh \left(\theta-\ln \left(T / T_{K}\right)\right)} \ln \left(1+e^{-\epsilon_{1}(\theta)}\right) \text {. }
$$

The Kondo result [9] is given by taking $m \rightarrow \infty$.

Let us now extract physical predictions from this. The easiest result to get is the cross-over exponent $\varphi$. To obtain it we observe, following [14 that the system (6) implies the periodicity of the pseudo-energies: $\epsilon_{j}[\theta+(m+1) i \pi]=\epsilon_{j}(\theta)$. As a consequence, close to $\theta=\infty$ we can expand

$$
\ln \left(1+e^{-\epsilon_{j}(\theta)}\right)=\sum_{k=0}^{\infty} L_{j}^{(k)}\left(e^{-2 \theta /(m+1)}\right)^{k}
$$

Moreover one can show by explicitly plugging the expansion into the equations (6) that the term $k=1$ vanishes identically. The $k=0$ term follows from the solution of the system

$$
x_{j}^{2}=\left(1+x_{j-1}\right)\left(1+x_{j+1}\right)
$$

where $x_{j} \equiv e^{-\epsilon_{j}(\infty)}$, yielding

$$
1+x_{j}=\left[\frac{\sin \pi(j+1) /(m+1)}{\sin \pi /(m+1)}\right]^{2} \text {. }
$$

The expansion (8) enables us to find the UV behaviour of $f_{\text {imp. }}$. As $T \rightarrow \infty$, the integral is dominated by the $\theta$ large region where the expansion is expected to be valid, so that

$$
\frac{f_{\mathrm{imp}}}{T}=-\frac{1}{2} \ln \left(1+x_{1}\right)+\sum_{k=2}^{\infty}\left(\frac{T_{K}}{T}\right)^{2 k /(m+1)} f_{U V}^{(k)}
$$

plus perhaps some non-universal bulk terms. Thus we recover the fact that the dimension of the energy operator at the special transition is $1-2 /(m+1)$. Standard finite-size and boundary scaling arguments imply that $f_{\text {imp }}$ is a function of $\left(x^{s}-x_{c}^{s}\right)^{\nu / \varphi} R$ where $\nu$ is the usual [5] thermal exponent, $\nu=\frac{m+1}{4}$. Also, from perturbation theory we expect that $f_{\text {imp }}$ is analytic in $x^{s}-x_{c}^{s}$. Therefore

$$
\frac{\varphi}{\nu}=\frac{2}{m+1}
$$


and $\varphi=1 / 2$ as desired [7]. Moreover we deduce from the absence of the $k=1$ term in the expansion (11) that the one-point function of the boundary energy vanishes at the UV fixed point, a known characteristic of the special transition [6]. In the $m \rightarrow \infty$ Kondo limit, the expansion results in the familiar log terms of the Kondo problem.

We also find a similar expansion near the free fixed point $(T \rightarrow 0)$. In this limit only the region $\theta$ negative and large contributes to the impurity free energy. One can thus expand out the $1 / \cosh$ in (7) as

$$
\frac{1}{2 \cosh \left(\theta-\ln \left(T / T_{K}\right)\right)} \approx \frac{T}{T_{K}} e^{-\theta}-\left(\frac{T}{T_{K}}\right)^{3} e^{-3 \theta}+\ldots
$$

We can integrate each piece individually because $\epsilon_{1} \approx \exp (-\theta)$ as $\theta \rightarrow-\infty$, yielding

$$
\frac{f_{\mathrm{imp}}}{T}=\sum_{k=1}^{\infty}\left(\frac{T}{T_{K}}\right)^{2 k-1} f_{I R}^{(k)}
$$

Notice that it goes to zero at $T=0$. The power of the first correction indicates that the dimension of the energy operator at the free fixed point is $1-(-1)=2$, as expected [6]. Moreover, by using perturbed conformal field theory, one in fact finds that the universal contribution to the $T^{2}$ term must vanish, as indeed seen in (11).

The crucial identification with the special transition can be obtained by looking at the evolution of the boundary " $g$-function" [15,16]. On a long strip of length $L$, the partition function with boundary conditions $A$ and $B$ at the sides reads $Z=g_{A} g_{B} \exp \left(-E_{0} L\right)$. Thus $g$ associated with our boundary condition should be $g=\exp \left(-f_{\text {imp }} / T\right)$. There are a number of subtleties which arise [17]; however, for massless theories these should result only in multiplying $g$ by an overall constant independent of scale. Thus from the TBA we see how $g$ flows from the UV (special) to IR (free), the expansions (11) and (12) giving

$$
\frac{g^{U V}}{g^{I R}}=\left(1+x_{1}\right)^{1 / 2}=\frac{\sin 2 \pi /(m+1)}{\sin \pi /(m+1)} .
$$

To derive $g^{U V}$ and $g^{I R}$ from conformal field theory, one needs to construct the boundary states in the manner of [15]. In the $O(n)$ model, this is problematic because of the non-locality; we discuss this below. However, without knowing these states explicitly we can still find the boundary "fusion" operator $\Phi$ which changes the boundary conditions from free to special. This enables us to determine the ratio $g^{U V} / g^{I R}$, and confirm the 
result numerically. If the boundary conditions $A$ and $B$ are such that only the state created by the operator $\Phi_{r, s}$ (and its descendants) propagate along the strip, it follows that for minimal models [15,16] that $g_{A} g_{B}=S_{1,1}^{r, s}$, where $S_{r^{\prime}, s^{\prime}}^{r, s}$ is the modular-transformation matrix for the minimal-model characters. We choose $A$ to be free boundary conditions, and $B$ such that $\Phi_{r, 1}$ propagates. We then change the boundary condition $A$ to the special boundary conditions by inserting the boundary operator $\Phi$. If $\Phi=\Phi_{1, s}$ for some $s$ then

$$
\frac{g^{U V}}{g^{I R}}=\frac{S_{1,1}^{r, s}}{S_{1,1}^{r, 1}}=\frac{\sin s \pi /(m+1)}{\sin \pi /(m+1)}
$$

Comparing with (13), we see that the TBA indicates that $\Phi=\Phi_{1,2}$. This is consistent with the Kondo case, where $\Phi$ is the $S U(2)_{1}$ primary field [18]. This field has bulk dimension $1 / 4$, which is the $m \rightarrow \infty$ limit of the dimension of $\Phi_{1,2}$ field.

To check this numerically, first recall that the surface exponents for the "fuseau" operators (operators which force $L$ lines down the strip) are $d_{L}^{s}=h_{L+1,1}$ [19]. It follows that if both $A$ and $B$ are free boundary conditions and $L$ lines go along the strip, the state that propagates corresponds to the operator $\Phi_{L+1,1}$. Thus if we change the boundary conditions on both sides of the strip from free to special, the operators in the OPE $\Phi \times$ $\Phi \times \Phi_{L+1,1}$ are those which propagate. For $\Phi=\Phi_{1,2}$, this means that $\Phi_{L+1,3}+\Phi_{L+1,1}$ propagate. It is easy to see that $h_{L+1,3}<h_{L+1,1}$ for $L \geq 1$. Hence the exponent of the $L$-lines fuseau operator at the special point should be $h_{L+1,3}$ while it is $h_{L+1,1}$ at the free point. It is easy to investigate this question numerically by explicitly diagonalizing the transfer matrix of the lattice $O(n)$ model. The question of surface exponents has been addressed in [19,20]. In [20] the authors study the adsorption transition for polymers but unfortunately they do not discuss the fuseau operators for $L>1$ nor the case $n \neq 0$. We have therefore extended their analysis to these cases. The results look as usual and confirm completely that $\Phi_{L+1,3}$ propagates down the strip at the special point, giving strong evidence indeed for our conjecture. We give some examples for $n=1$ below.

Exponents for polymers follow from the above by choosing $m=2$. Other quantities can be obtained by studying values near $m=2$ and taking appropriate derivatives. For instance the ratio (14) is of course equal to one for $n=0$ but the result for one polymer loop follows from the first non-trivial term: $g^{U V} / g^{I R}=2 / 3 \sqrt{3}$. The analysis of the free energy for non-integer values of $m$ is technically more complicated [13], but for the polymer 
one can obtain it as an analytic function of $R^{2 / 3}\left(x^{s}-x_{c}^{s}\right)$. One expects generally that it is proportional to the number of adsorbed monomers $N_{\text {ads }}$, and that

$$
N_{\mathrm{ads}}=N^{\varphi} F\left[\left(x^{s}-x_{c}^{s}\right) N^{\varphi}\right]
$$

where $F$ is a scaling function that would be most interesting to compute. What we obtained of course looks qualitatively like this, but instead of $N$ we have the variable $R$. By finitesize scaling, the typical length of the polymer on a cylinder of radius $R$ at critical bulk coupling varies as $N \propto R^{4 / 3}$, so using $\varphi=1 / 2$ our expansion (11) looks indeed like (15).

Equation (13) can also be continued to $n<0$. In this regime, $g^{U V}<g^{I R}$, so the " $g$ conjecture" [16] does not hold for non-unitary models, as was the case for the $c$-theorem of Zamolodchikov. Another interesting characteristic is that we find perfectly reasonable results at $n \geq 1$ too. The standard result [21] that the $O(n)$ model has a one-dimensional phase transition only for $n \leq 1$ can naively be used to argue that the special transition disappears for $n>1$. Think, however, of the lattice $O(n)$ model. The high temperature expansion of the Ising model gives an $O(n=1)$ model with a fugacity $x=\operatorname{th} \beta$ per monomer. The special transition in the loop model occurs at the value of fugacity for monomers at the boundary that corresponds to $\beta=\infty$, since the Ising model in one dimension orders only at zero temperature: $x_{c}^{s}=1$. Thus there $i s$ a special transition in the geometrical $O(n)$ model with $n=1$ although it is usually agreed that there is none for the Ising model. The Ising spin model is a subset of the geometrical model only. The geometrical objects experience a transition, but the geometrical properties do not have a local meaning in terms of spins. For $n>1$, it is possible that there is a finite transition for a finite boundary monomer fugacity which however would correspond to sending the boundary coupling of the $O(n)$ model into the complex plane. If so, one really could consider the spin degrees of freedom in the Kondo problem as the $n=2$ limit of the special transition of the $O(n)$ model. This certainly seems true from the formal point of view of the $S$ matrices and the TBA.

Measurements of the surface exponents at the special transition in the $n=1$ model are easier at this point because we know $x_{c}^{s}$ exactly; for other values of $n$ it must be determined numerically. We thus numerically diagonalize the transfer matrix at $n=1$ and find the gaps in the spectrum, which are proportional to the scaling dimensions. With special boundary conditions on both sides of a strip of width $M$ lattice sites, these dimensions are 


$\begin{array}{lllllll} & M=3 & 4 & 5 & 6 & \infty & \text { expected } \\ L=2 & .19106 & .17961 & .17340 & .16923 & .167(2) & 1 / 6 \\ L=3 & 1.19444 & 1.13747 & 1.10741 & 1.08955 & 1.02(2) & 1 \\ L=4 & 2.03612 & 2.75960 & 2.74731 & 2.72118 & 2.6(2) & 5 / 2\end{array}$

This confirms our identification of the $\Phi_{1,2}$ operator as the boundary fusion operator. We also find from the above results $g^{U V} / g^{I R}=\sqrt{2}$. This cannot really be interpreted in terms of spin. 2 Indeed, the IR fixed point is just free boundary conditions for the high temperature contours and therefore also for the Ising spins, hence $g^{I R}=1$ [15]. The UV fixed point corresponds to infinite coupling of Ising spins at the boundary, so these spins can be either all + or all - . Moreover, summing over these two choices leads essentially to free boundary conditions for the spins next to the boundary. Hence the UV fixed point should be interpreted as some superposition of (two possible) fixed and one free boundary condition. This can be made more quantitative by recalling that the one-point function of the energy operator vanishes at the special transition. This happens for a boundary state invariant under duality, for example the superposition $\frac{1}{2}(\mid$ fixed +$\rangle+\mid$ fixed -$\rangle+\sqrt{2} \mid$ free $\left.\rangle\right)$. There is no such boundary state in the Ising spin model since the coefficients are not integers. However, there can be such a state in the non-minimal loop model where the condition of integrality is relaxed [22].

We have concentrated on the flow towards the desorbed phase, but the flow towards the adsorbed one should not be very different since once a polymer is adsorbed at the boundary and has fractal dimension one, the other ones see free boundary conditions on a slightly smaller system. We have checked numerically that in the adsorbed phase the fuseau exponents again have the value $d_{L}^{s}=h_{L+1,1}$.

Acknowledgments: This work was supported by the Packard Foundation, the National Young Investigator program (NSF-PHY-9357207) and the DOE (DE-FG03-84ER40168). We thank P. Dorey for useful correspondence.

2 We do note, however, that up to a constant shift the free energy at $n=1$ should also describe the flow from free to fixed boundary conditions in the Ising model [16], which has precisely this ratio. This case corresponds to perturbation by the magnetic field, which is not invariant under spin flip and is not equivalent to our problem. However, one can check the dimensions of the magnetic field at the free and fixed points, and one finds that they are the same as those of the energy operator at the special and free points, respectively. 


\section{References}

[1] For a partial survey, see I. Affleck, "Conformal Field Theory Approach to Quantum Impurity Problems", UBCTP-93-25, cond-mat/9311054.

[2] R. Villanove and F. Rondelez, Phys. Rev. Lett. 45 (1980) 1502.

[3] P.G. De Gennes, Scaling concepts in polymer physics, Cornell University Press (1979).

[4] K. Binder, in Phase transitions and critical phenomena, Vol. 8, ed. by C. Domb and J. Lebowitz, Academic Press (1983).

[5] B. Nienhuis, J. Stat. Phys. 34 (1984) 731, and references therein.

[6] T. Burkhardt and J. Cardy, J.Phys. A20 (1987) L233.

[7] T. Burkhardt, E. Eisenreigler and I. Guim, Nucl. Phys. B316 (1989) 559.

[8] S. Ghoshal and A.B. Zamolodchikov, "Boundary State and Boundary $S$ Matrix in Two-Dimensional Integrable Field Theory", RU-93-20, hep-th/9306002.

[9] N. Andrei, K. Furuya, and J. Lowenstein, Rev. Mod. Phys. 55 (1983) 331;

A.M. Tsvelick and P.B. Wiegmann, Adv. Phys. 32 (1983) 453.

[10] P. Fendley, Phys. Rev. Lett. 71 (1993) 2485, cond-mat/9304031.

[11] A.B. Zamolodchikov, Mod. Phys. Lett. A6 (1991) 1807.

[12] F. Smirnov, Phys. Lett. B275 (1992) 109.

[13] P. Fendley and H. Saleur, Nucl. Phys. B388 (1992) 609, hep-th/9204094.

[14] Al.B. Zamolodchikov, Nucl. Phys. B358 (1991), 524.

[15] J. Cardy, Nucl. Phys. B324 (1989) 581.

[16] I. Affleck and A. Ludwig, Phys. Rev. Lett. 67 (1991) 161.

[17] P. Fendley and H. Saleur, "Deriving Boundary $S$ Matrices", USC-94-001, hepth/9402045.

[18] I. Affleck and A. Ludwig, Nucl. Phys. B352 (1991) 849; Nucl. Phys. B360 (1991) 641.

[19] B. Duplantier and H. Saleur, Phys. Rev. Lett. 57 (1986) 3179.

[20] I. Guim and T. Burkhardt, J. Phys. A22 (1989) 1131.

[21] R. Balian and G.Toulouse, Ann. Phys. 83 (1974) 28.

[22] P. Di Francesco, H. Saleur and J.B. Zuber, J. Stat. Phys. 49 (1987) 57. 